\title{
Axonal phenotype of Charcot-Marie-Tooth disease associated with a mutation in the myelin protein zero gene
}

\author{
F Chapon, P Latour, P Diraison, S Schaeffer, A Vandenberghe
}

Laboratoire de

Neuropathologie

F Chapon

Service de Neurologie

Dejerine, Centre

Hospitalier

Universitaire de Caen,

14033 Caen, France

P Diraison

S Schaeffer

Unité de

Neurogénétique

Moléculaire, Hospices

Civils de Lyon, Hôpital

de l'Antiquaille 69005

Lyon, France

P Latour

A Vandenberghe

Correspondence to:

Dr F Chapon, Laboratoire

de Neuropathologie, CHRU

de CAEN, Avenue Côte de

Nâcre, F-14033 Caen Cedex,

France. Telephone 0033231

064621 ; fax 003323106

46 27; email

chapon-f(a)chu-caen.fr

Received 23 July 1998 and in revised form

16 November 1998

Accepted 20 November 1998

\begin{abstract}
A French family had Charcot-Marie-Tooth disease type 2 (CMT2) which was characterised by late onset of peripheral neuropathy involvement, Argyll Robertsonlike pupils, dysphagia, and deafness. Electrophysiological studies and nerve biopsy defined the neuropathy as axonal type. Genetic analysis of myelin protein zero (MPZ) found a mutation in codon 124 resulting in substitution of threonine by methionine. One of the patients, presently 30 years old, showed only Argyll Robertson-like pupils as an objective sign but no clinical or electrophysiological signs of peripheral neuropathy.

(F Neurol Neurosurg Psychiatry 1999;66:779-782)
\end{abstract}

Keywords: Charcot-Marie-Tooth; myelin protein zero; axonal neuropathy; Argyll-Robertson syndrome

Charcot-Marie-Tooth disease (CMT) is a group of clinical, paraclinical and genetic heterogeneous disorders affecting the peripheral nervous system. CMT type 1 (or CMT1)

\section{Family Ly135}
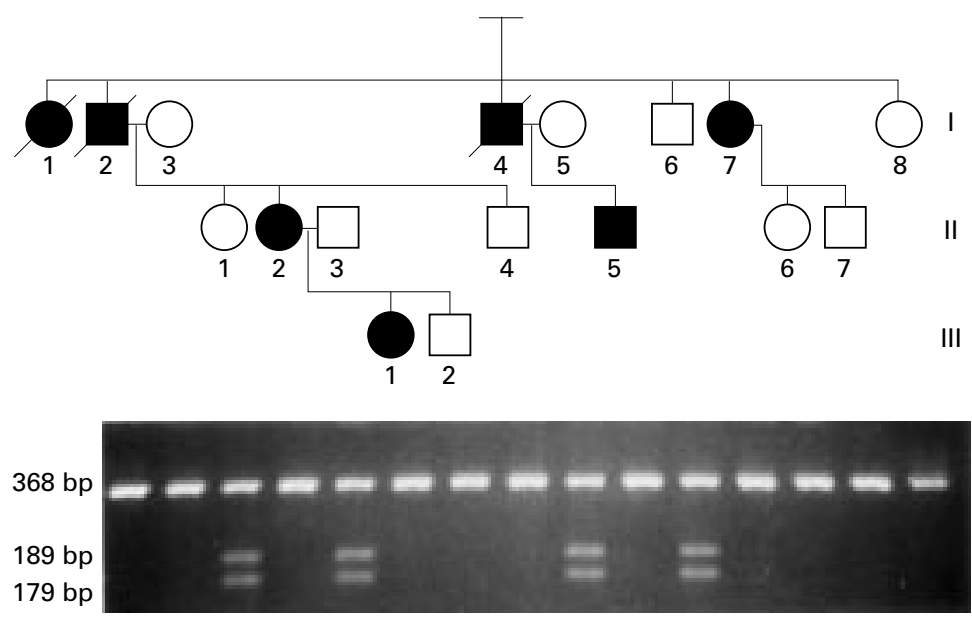

Figure 1 (Top) Pedigree of family Ly135 with a mutation in MPZ. Carriers of mutation are represented by a filled symbol, unaffected subjects by an open symbol. (Bottom) Polymerase chain reaction (PCR) analysis: the mutation creates a restriction site cleaved by the enzyme Hsp92II. The sample on the right is a control sample of a healthy unrelated person. The normal PCR product is 368 nucleotides in length. Digested fragments are 189 and $179 \mathrm{bp}$ long. is the "demyelinating" form of the disease with onion bulb formation due to demyelination and remyelination, and severely reduced nerve conduction velocities (NCVs) in the motor and sensory nerves. CMT2 is the "axonal" form with no segmental demyelination on nerve biopsies and normal or subnormal NCVs. CMT1 and CMT2 can be differenciated on the basis of motor median NCVs of 38 $\mathrm{m} / \mathrm{s} .{ }^{1}$ Mutations in the myelin protein zero (MPZ) gene have been associated with CMT $1 .^{2}$ Different phenotypes have been associated with different MPZ mutations ${ }^{3}$ including CMT 1B with onset within the first two decades of life, Dejerine-Sottas syndrome with onset in early childhood, and congenital hypomyelination. For CMT2, three candidate gene loci are known ${ }^{2}$ : 1p36, 3q13-q22, and 7p14. We here report the occurrence of a CMT2 family with a mutation in the MPZ gene hitherto involved in CMT1. Moreover, the family we describe shows very peculiar clinical features.

\section{Materials and methods}

CLINICAL AND PATHOLOGICAL EVALUATION

The family reported herein originates from Normandy (western France) and includes three generations (fig 1). Based on family history, the mother and maternal grandfather of the first generation were known to be affected by a peripheral neuropathy. Clinical data were obtained in all patients and relatives. Electrophysiological investigation was performed on six living subjects (I.7, II.2, II.5, II.7, III.1, and III.2) as well as in one subject now deceased (I.1). Furthermore, two subjects also underwent a sural nerve biopsy (I.1 and II.2). Light microscopic and ultrastructural examination were done according to standard procedures. Study of teased fibres was performed on case II.2, according to the classification proposed by Dyck et al. ${ }^{4}$

\section{GENETIC STUDIES}

DNA from all living family members was analysed. Exon sequences of MPZ were subjected to single strand conformational polymorphism analysis. Primer sequences, PCR amplification, and sequencing were performed as described before. ${ }^{5}$ PCR fragments were digested with 


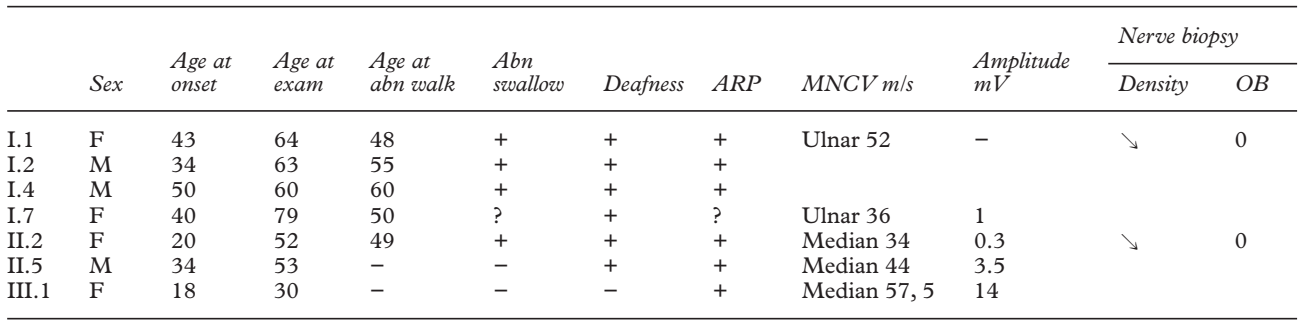

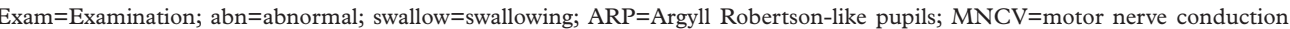
velocity; $\mathrm{OB}=$ onion bulb; $0=$ absence; $\searrow=$ lowered.

Normal values are: $\mathrm{NCV}>48 \mathrm{~m} / \mathrm{s}$; median nerve amplitude $>5 \mathrm{mV}$; ulnar nerve amplitude $>6 \mathrm{mV}$.

restriction enzyme Hsp92II according to the manufacturer's protocol (Promega Corp, Madison, WI, USA).

\section{Results}

CLINICAL, ELECTROPHYSIOLOGICAL, AND PATHOLOGICAL INVESTIGATIONS

Data from the seven affected subjects (four out of six in the first generation, two out of six in the second generation, and one out of the third generation) are reported in the table. Although three of them (I.1, I.2, I.4) were deceased at the time of the present study, they could none the less be identified as being affected by a peripheral neuropathy, according to their medical history. One of them with an association of CMT and Argyll-Robertson-like pupils was reported earlier. ${ }^{6}$

The pedigree is consistent with the transmission of an autosomal dominant trait.

\section{Limbs}

Onset of peripheral neuropathic symptoms was between the fourth and the sixth decades of life in five out of the seven patients investigated; the two remaining patients (II.2 and III.1) showed an earlier onset, with lightning pains as first symptom. All the patients, except case III.1, who is presently only 30 years old, gradually developed atrophy and weakness of the distal muscles, firstly in the legs, secondly, in the upper limbs. Major disability in walking was noted around the 50 s or later. Patient II. 2 is more severely disabled, using a wheelchair since the age of 49 . Sensation was altered with unsteady gait and frequent complaints of pain early in the evolution. Trophic changes, as purplish discolouration of the skin, oedema, coolness, and more rarely plantar ulcers, were sometimes seen. Six patients were deaf.

Argyll-Robertson-like pupils were present in all the affected patients except patient I.7 in whom this sign, not noticed in youth, could not be detected as the patient showed glaucoma and cataract at the time of examination. It should be noted that Argyll-Robertson-like syndrome was the only objective symptom in patient III.1.

Difficulty in swallowing occurred in four of the patients and in patient I.2 it resulted in choking with aspiration pneumonia and subsequent death.

Electrodiagnostic studies showed normal or moderately slowed motor nerve conduction velocities (MNCVs) but very low motor compound muscle action potential (CMAP) amplitudes and also decreased sensory potential amplitudes (measured in patient II.2). These findings, consistent with the diagnosis of axonal neuropathy, were found in all patients investigated except in patient III.1; in this patient, both amplitudes and velocities were normal as well as recruitment.

Nerve biopsy, performed in patients I. 1 and II. 2 only, did not disclose any sign of demyelination and remyelination (onion bulbs) but showed depopulation and rare clusters of small myelinated fibres (fig 2A, C). Examination of teased fibres, performed in patient II.2, disclosed some specimens of type E fibres (fig 2 B), typical for axonal degeneration. ${ }^{4}$

\section{GENETIC ANALYSIS}

Screening the MPZ gene for the presence of SSCPs showed an aberrant electrophoretic migration profile of exon 3. Sequencing disclosed a mutation in codon 124 , resulting in substitution of threonine by methionine (ACG->ATG). This mutation creates a Hsp92II and abolishes an MaeII restriction enzyme site and study of transmission in the family showed concordance with ArgyllRobertson-like pupils and CMT in all patients except patient III.1 (fig 1). In this patient, who also had the mutation, Argyll-Robertson-like pupils was the only objective sign of the disease.

\section{Discussion}

We studied a CMT2 family in which a mutation in MPZ was found. The complete clinical presentation was a sensorimotor neuropathy with trophic changes, but several points have to be emphasised: a late onset was found in most of the patients. However, the recorded age of onset may be delayed in relation to exact onset due to the lack of objective signs of neuropathy; indeed, walking disability caused by weakness in the limbs only started around the age of 50 . When the disease was found earlier (patients II.2 and III.1), diagnosis was based only on subjective signsfor example, pains, possibly with ArgyllRobertson-like pupils. Indeed, these were the only abnormalities shown by patient III.1, presently 30 years old.

Argyll-Robertson-like pupils associated with CMT has been previously documented. ${ }^{6}$ One of the cases reported by these authors belongs to the family described here, (patient I.1), and diagnosed as a patient with neural type CMT with Argyll-Robertson-like pupils. In another 

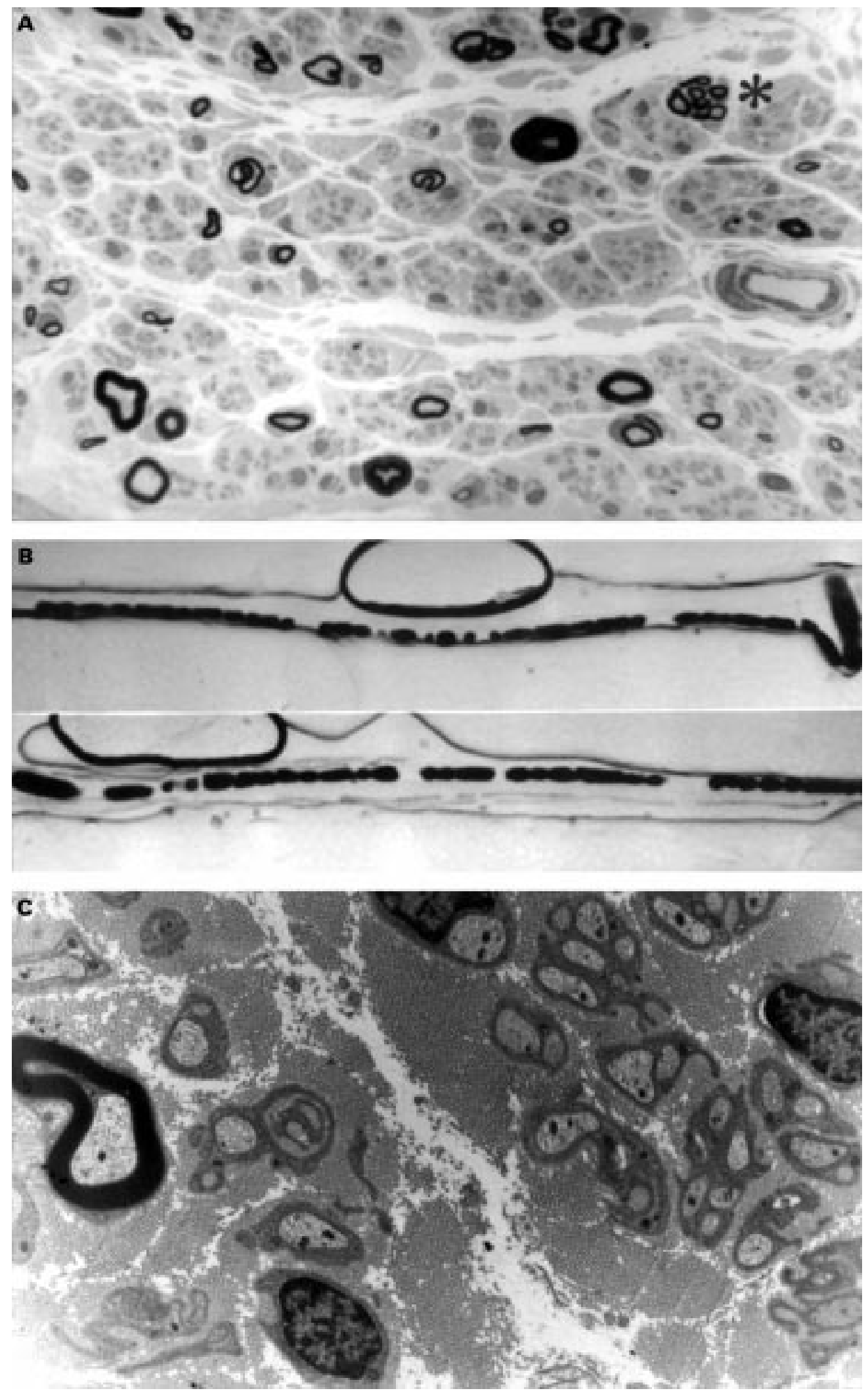

Figure 2 Sural nerve biopsy of patient II.2. (A) A dramatic loss of myelinated fibres with rare clusters of small myelinated fibres $\left(^{\star}\right)$ are seen in a semithin section with thionin staining (magnification $\left.\times 840\right)$. No onion bulb-like structures are seen. (B) Teased fibres show myelin ovoids (magnification $\times 170$ ). (C) Ultrastructural study (magnification $\times 4800$ ) shows no onion bulbs, no abnormal Schwann cell processes, and no abnormal compaction of myelin sheaths.

family, with association of neural CMT and Argyll-Robertson-like pupils, ${ }^{7}$ absence of linkage to a candidate region for CMT2 in 1 p35-36 was reported..$^{8}$ It would be of interest to re-examine such families for the presence of mutations in the MPZ gene.

Another peculiar clinical sign, characteristic of this family, is the occurrence of deafness, a 
deficiency that was present in all patients but one (patient III.1). The age of onset of this symptom could not, however, be precisely determined in this retrospective study except in patient II.5, in whom loss of hearing was noted from the age of 44 .

Finally, disability in swallowing is another characteristic of the disease; it could be severe in some patients and even lead to death due to choking as in patient I.2.

According to electrophysiological and histological studies, this type of hereditary neuropathy was classified as axonal. Electrophysiological measurement of median MNCVs has been a valuable tool in differentiating between CMT1 and CMT2 forms. A discriminatory value of $38 \mathrm{~m} / \mathrm{s}$ has been postulated with the lowered velocities for the CMT1 form. ${ }^{1}$ Because two members of the family had median MNCVs above this value, they were classified as CMT2; one of them is paucisymptomatic (pains only), but the other one has classic clinical features of CMT. In the patient with median MNCVs $<38 \mathrm{~m} / \mathrm{s}$ (II.2), CMAPs were very low as well as the sensory potential amplitudes. These electrophysiological characteristics are consistent with axonal lesions. ${ }^{9}$

Although mutations in MPZ have been previously associated with CMT1, with very low MNCVs, usually $<25 \mathrm{~m} / \mathrm{s}$, mutations in MPZ cover a large range of phenotypes including severe congenital hypomyelination, DejerineSottas syndrome, CMT1B with lowered MNCVs, and CMT2 with normal or subnormal MNCVs. Recently, Marrosu et $a l^{10}$ reported another mutation in the MPZ gene also resulting in axonal CMT as shown by median MNCV. The patients in their studied family present a typical CMT clinical phenotype, but without the very particular signs we report here (deafness, Argyll-Robertson-like pupils, and dysphagia).

In an earlier report, ${ }^{11}$ we described a mutation of codon 122, close to codon 124. According to a molecular model for the extramembraneous part of the protein, amino acid 122 should be located at the base of the molecule close to the Schwann cell membrane. ${ }^{12}$ The phenotype associated with this mutation was mild with late onset with NCVs between 20 and $40 \mathrm{~m} / \mathrm{s}$. No sign of deafness or abnormal pupillary response was found. To date, the same mutation in codon 124 has been described in two other reports ${ }^{13}{ }^{14}$ in which the patients are poorly documented and classified as mild, late onset CMT1 types. It is highly probable that the occurrence of this mutation has so far been underestimated due either to an inaccurate diagnosis of mild cases or to the fact that the MPZ gene is not routinely screened for mutations in patients with CMT2. It is possible that the mutation of codon 124 affects a structure specific for the ciliar nerves ${ }^{15}$ thereby causing Argyll-Robertson-like pupils. However, Argyll-Robertson-like pupils are not specific for phenotypes associated with MPZ mutations.

We thank Professor Lapresle and Professor Cambier who provided clinical data from their patients, and S Gamon and personnel of the Biochemistry laboratory, Hôpital de personnel of the Biochemistry laboratory, Hopital de supported by the Association Française contre les Myopathies supported by the Association Française contre les Myopathies
and the Hospices Civils de Lyon. Our laboratories are members and the Hospices Civils de Lyon. Our laboratories are members of the European CMT Consortium sponsored by
Biomed 2 grants No CT961614 and No CT960055.

1 Harding AE, Thomas PK. The clinical features of hereditary motor and sensory neuropathy types I and II. Brain 1980;103:259-80.

2 De Jonghe P, Timmerman V, Nelis E, et al. Charcot-MarieTooth disease and related peripheral neuropathies. fournal of the Peripheral Nervous System Nervous Syst 1997; 2:370-

3 Warner LE, Hilz MJ, Appel SH, et al. Clinical phenotypes of different MPZ (P0). Mutations may include CharcotMarie-Tooth type 1B, Dejerine-Sottas, and congenital hypomyelination. Neuron 1996;17:451-60.

4 Dyck PJ, Giannini C, Lais A. Pathologic alterations of nerves. In: Dyck PJ, Thomas PK, Griffin GW, et al, eds. Peripheral neuropathy. Philadelphia: Saunders 1993: $514-$ 595.

5 Nelis E, Timmerman V, De Jonghe P, et al. Rapid screening of myelin genes in CMT1 patients by SSCP analysis: identification of new mutations and polymorphisms in the P0 gene. Hum Genet 1994;94:653-7.

6 Salisachs P, Lapresle J. Argyll-Robertson-Like pupils in the neural type of Charcot-Marie-Tooth disease. Eur Neurol 1977;16:172-5.

7 André-Van Leeuwen M. De la valeur des troubles pupillaires en dehors de la syphilis comme signe précoce ou forme frustre d'une affection hérédo-dégénérative. III. Une souche d'amyotrophie de Charcot-Marie-Tooth présentant des troubles pupillaires chez un grand nombre de ses membres et, chez l'un d'entre eux, des lésions typiques de névrite hypertrophique. La famille Smits. Monatsschrift für Psychiatrie und Neurologie 1943;108:1-89.

8 Timmerman V, De Jonghe P, Spoelders P, et al. Linkage and mutation analysis of Charcot-Marie-Tooth neuropathy type 2 families with chromosomes 1p35-36 and Xq13. Neurology 1996;46:1311-8 Monatsschrift für Psychiatrie and Neurologie

9 Research criteria for diagnosis of chronic inflammatory demyelinating polyneuropathy (CIDP). Report from an Ad Hoc Sub Committee of the American Academy of Neurology AIDS Task Force. Neurology 1991;41:617-8.

10 Marrosu MG, Vaccargiu S, Marrosu G, et al. CharcotMarie-Tooth disease type 2 associated with mutation of the myelin protein zero gene. Neurology 1998;50:1397-401.

11 Blanquet-Grossard F, Pham-Dinh D, Dautigny A, et al. Charcot-Marie-Tooth type 1B neuropathy: a mutation at the single glycosylation site in the major peripheral myelin the single glycosylation site in the major peript
glycoprotein P0. Hum Mutat 1996;8:185-6.

12 Kirschner DA, Szumowski K, Gabreëls-Festen AAWM, et al. Inherited demyelinating peripheral neuropathies: relating myelin packing abnormalities to P0 molecular defects. $\mathcal{F}$ Neurosci Res 1996;46:502-8.

13 Wolf C, Arnold H, Reichenbach H, et al. Screening of myelin gene in CMT 1 patient without duplication in chromosomal region 17p11.2-p12. Fournal of the Peripheral Nervous System 1997;2:402.

14 Schiavon F, Rampazzo A, Merlini L, et al. Mutations of the same sequence of the myelin P0 gene causing two different phenotypes. Hum Mutation 1997; Mutation in brief No 86 Online.

15 Garcin R, Gruner J, Man HX. Etude pathogénique du signe d'Argyll-Robertson dans la névrite hypertrophique de Dejerine-Sottas. Document anatomo-clinique. Presse Méd 1960;10:357-60. 